\title{
ESTUDO DA ADIÇÃO DA LIGNINA KRAFT NAS PROPRIEDADES MECÂNICAS DOS BRIQUETES DE RESÍDUOS DA INDÚSTRIA MOVELEIRA
}

\section{STUDY OF THE ADDITION OF LIGNIN EXTRACTED FROM KRAFT BLACK LIQUOR IN THE MECHANICAL PROPRIETIES OF BRIQUETTES}

\author{
Adriana de Fátima Gomes Gouvêa ${ }^{1}$ Ana Márcia Macedo Ladeira Carvalho ${ }^{2}$ \\ Cláudio Mudadu Silva ${ }^{3}$ Angélica de Cássia Oliveira Carneiro ${ }^{2}$ \\ Paulo Fernando Trugilho ${ }^{4}$ Fabiana Paiva de Freitas ${ }^{5} \quad$ Lumena Boroni Valadares $^{6}$ \\ Claudia Marcia Gomes ${ }^{7}$ Emerson Belarmino Costa ${ }^{8}$
}

\begin{abstract}
RESUMO
A fim de melhorar a eficácia global da produção de energia, uma estratégia é o modelo de biorrefinarias, em que todos os componentes de biomassa são totalmente utilizados para produzir uma gama de produtos de maior valor agregado. A lignina kraft é um dos produtos oriundos do processo de produção de celulose e que pode ser utilizado na confecção de materiais densificados. O objetivo deste trabalho foi avaliar o comportamento da adição da lignina extraída do licor negro kraft em briquetes confeccionados com resíduos da indústria moveleira na forma de maravalha e partículas de painéis; em diferentes proporções. Os briquetes foram produzidos em briquetadeira laboratorial, usando as temperaturas de 60,75 e $90{ }^{\circ} \mathrm{C}$. O tempo de compactação e resfriamento foi de 5 minutos e pressão $68,9 \times 10^{5} \mathrm{~N} \cdot \mathrm{m}^{-2}$ (1000 PSI). A qualidade dos briquetes foi avaliada em função da determinação da propriedade mecânica. Determinou-se a carga de ruptura dos briquetes. A adição de lignina kraft aos resíduos de madeira nas proporções e temperatura adequadas apresentou vantagens de aumento de resistência mecânica dos briquetes. A lignina kraft apresentou vantagens como aglutinante na produção de briquetes de resíduo de madeira na temperatura de compactação de $90{ }^{\circ} \mathrm{C}$ com adição de $20 \%$ de lignina kraft e temperaturas de 60 e $75{ }^{\circ} \mathrm{C}$ de compactação na proporção de adição de 40 e $60 \%$ de lignina kraft, respectivamente.
\end{abstract}

Palavra-chave: briquetes; aglutinante; resíduo indústria moveleira.

1 Engenheira Florestal, $\mathrm{Dr}^{\mathrm{a}}$., Professora do curso de Engenharia Florestal, Universidade Estadual do Mato Grosso do Sul, Rod MS 450 Graziela Maciel Barroso Km 12, Campus Universitário, CEP 79200-000, Aquidauana (MS), Brasil.agouvea@uems.br

2 Engenheira Florestal, Dr ${ }^{\mathrm{a}}$., Professora do Departamento de Engenharia Florestal, Universidade Federal de Viçosa, Av. P. H Rolfs, s/n, Campus Universitário, CEP 36570-000, Viçosa (MG), Brasil. ana.marcia@ufv.br; cassinhacarneiro1@gmail.com

3 Engenheiro Civil, Dr., Professor do Departamento de Engenharia Florestal, Laboratório de Celulose e Papel, Universidade Federal de Viçosa, Av. P. H Rolfs, s/n, campus universitário, CEP 36570-000, Viçosa (MG), Brasil. mudado@ufv.br

4 Engenheiro Florestal, Dr., Professor do Departamento de Ciências Florestais, Laboratório de Tecnologia da Madeira, Universidade Federal de Lavras, Campus universitário, CEP 37200-000, Lavras (MG), Brasil. trugilho@dcf.ufla.br

5 Engenheira Florestal, Mestranda em Ciências Florestais, Departamento de Engenharia Florestal, Universidade Federal de Viçosa, Av. P. H. Rolfs, s/n, CEP 36570-000, Viçosa (MG), Brasil. fabianapf@hotmail.com

6 Engenheira Florestal, Departamento de Engenharia Florestal, Universidade Federal de Viçosa, Av. P. H. Rolfs, s/n, CEP 36570-000, Viçosa (MG), Brasil. lumenaboroni@hotmail.com

7 Engenheira Florestal, Dr ${ }^{\mathrm{a}}$. Professora do curso de Engenharia Florestal.Universidade Federal do Recôncavo da Bahia, R. Rui Barbosa, n 710, CEP: 44380-000, Cruz das Almas ( BA), Brasil. claudia-davi@hotmail.com

8 Graduando em Engenheira Florestal, Universidade Estadual do Mato Grosso do Sul, Rod MS 450 Graziela Maciel Barroso Km 12, Campus Universitário, CEP 79200-000, Aquidauana (MS), Brasil. emersonbelarmino@hotmail.com 


\begin{abstract}
In order to improve global effectiveness of energy production, a strategy is the model of bio-refineries in which all the components of biomass are totally used to produce a range of products with aggregated value. The kraft lignin is one of the products originated in the process of cellulose production that can be used in making densified products. The objective of this work was to evaluate the behavior of the addition of extracted kraft lignin of black kraft liquor in briquette manufactures with furniture industry waste, in the

form of shavings and panel particles in different proportions. The briquettes were produced in laboratory briquetting, using the temperatures of 60,75 and $90{ }^{\circ} \mathrm{C}$. The pressing and cooling time was 5 minutes and pressure $68,9 \times 10^{5} \mathrm{~N} \cdot \mathrm{m}^{-2}$ (1000 PSI). The briquette quality was evaluated through determining the mechanical properties. The kraft lignin in adequate proportions and temperature showed advantages in the increase of resistance and the durability of the briquettes. The kraft lignin presented advantages such as agglutinative in briquette productions from wood waste at a pressing temperature of $90{ }^{\circ} \mathrm{C}$ with addition of $20 \%$ of kraft lignin, and at pressing temperatures of 60 and $75{ }^{\circ} \mathrm{C}$ at a proportion of addition of 40 and $60 \%$ of kraft lignin to wood waste, respectively.
\end{abstract}

Keywords: briquetting; agglutinative; furniture industry.

\title{
INTRODUÇÃO
}

A atividade biológica produz 300 bilhões de toneladas por ano de biomassa vegetal, pela fotossíntese. A celulose e a lignina são os constituintes mais importantes e, por consequência, os compostos naturais mais abundantes na terra (BELGACEM; BLAYO; GANDINI, 2004).

Os produtos químicos desenvolvidos a partir da biomassa são os que possuem maior potencial em agregar valor a uma cadeia produtiva agroenergética em função da participação estratégica da indústria química no fornecimento de insumos e produtos finais a diversos setores da economia, como: petroquímico, farmacêutico, automotivo, construção, agronegócio, cosméticos, etc. Biocombustíveis e materiais estão em um segundo patamar de valoração, seguidos por energia e insumos químicos, como fertilizantes e defensivos agrícolas. No Brasil, esforços têm sido feitos de modo a se avaliar o potencial econômico da biomassa. Porém, o número de trabalhos voltados exclusivamente para o aproveitamento de resíduos agroindustriais na química é pequeno no País, excetuando-se aqueles voltados à obtenção de etanol de segunda geração e uso de efluentes, como a vinhaça (VAZ JUNIOR, 2012).

Resíduos e coprodutos de cadeias como a do etanol, biodiesel e florestas foram avaliados quanto ao seu potencial econômico, de modo a apresentar um panorama atual de perspectivas e de desafios a serem superados para sua incorporação à crescente economia verde nacional, além da sua contribuição para o desenvolvimento de uma química renovável e sustentável (VAZ JUNIOR, 2012).

Nos últimos anos, tem surgido um grande interesse mundial no desenvolvimento de tecnologias "verdes" que possibilitam a utilização de produtos de menor impacto ambiental. A química "verde", como um todo, implica no desenvolvimento de processos químicos e produtos que levem a um ambiente mais limpo, saudável e sustentável (WARNER; CANNON; DYE, 2004). Dentre as pesquisas nesta área, as que buscam a aplicação de recursos naturais na preparação dos materiais vêm crescendo, podendo-se destacar o uso de lignina kraft para diversas aplicações, como, por exemplo, resinas fenólicas e péletes. A adição de lignina à resina fenólica pode ser usada como um extensor, por exemplo, para aumentar a taxa de cura, reduzir custos ou para modificação química da resina, sendo que a lignina é quimicamente incorporada a resina fenólica. A lignina pode ser adicionada no início, durante o processo de aquecimento ou no final da reação de condensação (DUNKY; PIZZI; LEEMPUT, 2002). Na indústria alimentícia, a lignina é usada como aglutinante para melhorar a qualidade de péletes na alimentação de animais (KALIYAN; MOREY, 2009) e também para geração de biocombustíveis (PER TOMANI; SVÄRD; ÅMAND, 2008). Entretanto, estudos da aplicação da lignina kraft no processo de briquetagem são escassos (LURII, 2008), bem como informações sobre as propriedades dos briquetes.

Desse modo, o principal objetivo deste trabalho foi avaliar a qualidade dos briquetes confeccionados com resíduos da indústria moveleira, na forma de maravalha e partículas de painéis, com a adição de lignina 
extraída do licor negro kraft, em diferentes proporções de mistura e temperaturas de compactação, por meio de suas propriedades mecânicas.

\section{MATERIAL E MÉTODOS}

\section{Material}

Utilizou-se neste estudo três tipos de resíduos, a saber: resíduos de madeira de diversas espécies, provindos de serrarias, na forma de maravalha; resíduos de painéis compostos por aparas de MDP (Medium Density Particleboard) e MDF (Medium Density Fiberboard), provenientes de madeira de Pinus, ambos com acabamento melamínico de Baixa Pressão (tipo BP), misturados na proporção de 1:1 e a lignina extraída do licor negro, oriundo do processo Kraft.

\section{Métodos}

\section{Preparação do material}

Devido à desuniformidade dos resíduos provenientes dos painéis de MDF e MDF, foi necessário adequar a granulometria. Utilizou-se um moinho martelo para moagem. Posteriormente, as partículas, tanto de maravalha quanto de resíduo de painéis foram classificadas em peneira com malha 5,67 e 2,77 $\mathrm{mm}^{2}$. As partículas de painéis (MDP e MDF) por conterem adesivos ureia formaldeído e acabamento melamínico, foram misturados na proporção 1:1.

As partículas foram colocadas em uma câmara climática a $20^{\circ} \mathrm{C}$ e $65 \%$ de umidade relativa até atingir massa constante. $\mathrm{O}$ teor de umidade foi calculado de acordo a norma ABNT NBR 9484 (1986).

A lignina foi obtida a partir do licor negro kraft de eucalipto, na forma sólida (GARCÍA; MARTÍN; RODRÍGUEZ, 1984; NORTHEY, 1992). Posteriormente moída e peneirada, sendo recolhida a fração que ficou retida na peneira de 100 mesh (GOUVÊA, 2013).

\section{Produção dos briquetes}

Os briquetes foram produzidos em briquetadeira laboratorial, da marca Lippel, modelo LB-32, em três temperaturas 60,75 e $90{ }^{\circ} \mathrm{C}$, tempo de compactação de 5 minutos, tempo de resfriamento de 5 minutos e pressão de compactação igual a $68,9 \times 10^{5} \mathrm{~N} \cdot \mathrm{m}^{-2}$ (1000 PSI). A massa de partículas utilizada para produção de cada briquete foi de 16 gramas. Para a confecção dos briquetes, foram adicionadas 20,40 e 60\% de lignina kraft sobre a massa total de partículas da composição.

As condições de briquetagem foram definidas experimentalmente a partir de testes preliminares de tempo de compactação, tempo de resfriamento e temperatura. As condições escolhidas foram aquelas nas quais se obtiveram briquetes sem rachaduras ou fissuras e com menor deformação.

\section{Propriedade mecânica: carga de ruptura}

A carga de ruptura foi determinada empregando-se a máquina de ensaio universal modelo EMIC, na qual os briquetes foram comprimidos a uma velocidade de $3,5 \mathrm{~mm} \cdot \mathrm{min}^{-1}$ até a ruptura. A carga máxima de ruptura do briquete foi obtida e registrada através do software pav test. Utilizou-se a metodologia adaptada da norma ABNT NBR ISO $11093-9$ (2009), onde o briquete foi testado na posição vertical, sendo os esforços submetido no sentido paralelo as partículas.

\section{Delineamento experimental}

$\mathrm{Na}$ Figura 1 é apresentado o desenho esquemático do delineamento experimental utilizado para avaliação dos efeitos da temperatura e da proporção de adição de lignina kraft (LK) extraída do licor negro 

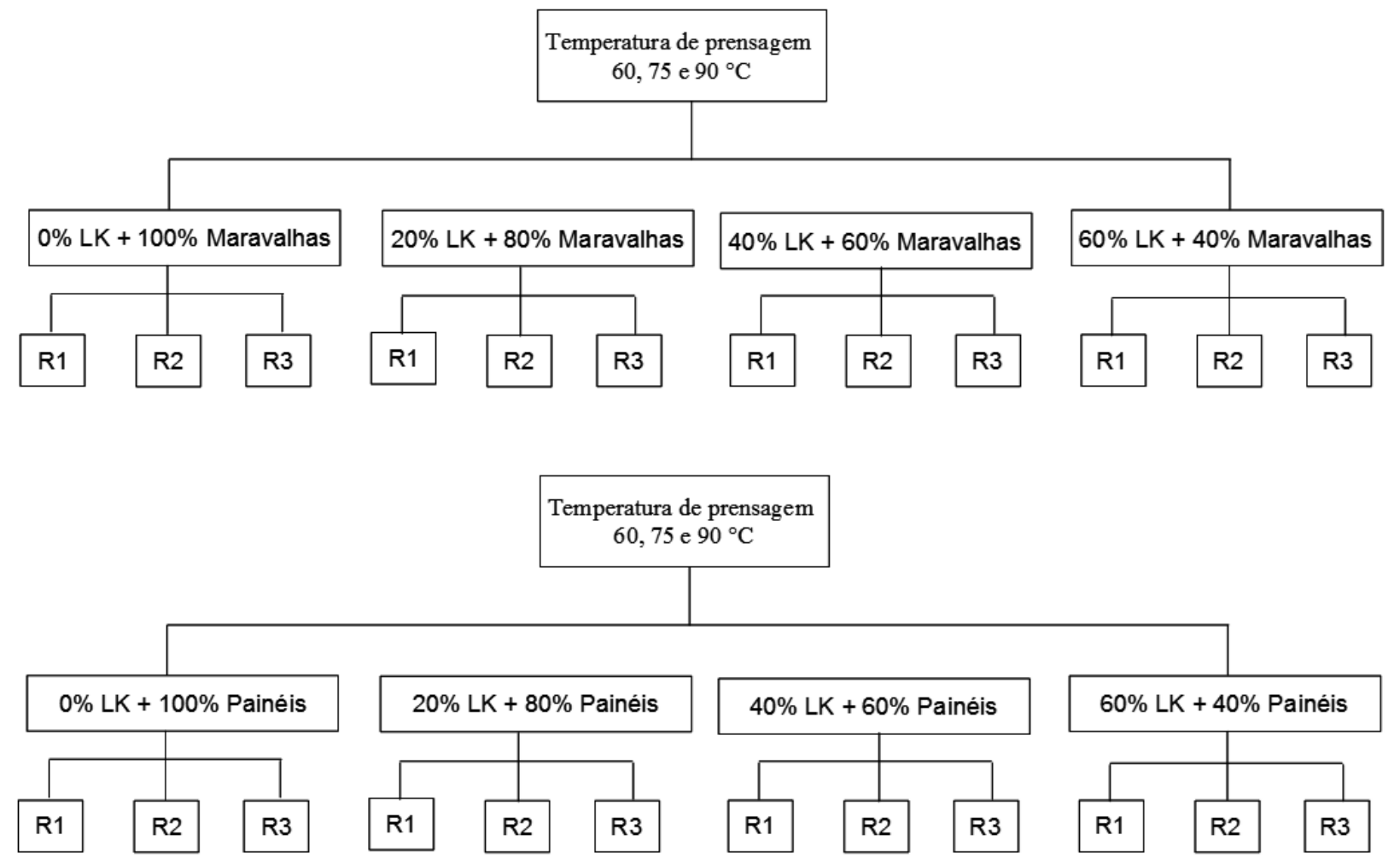

FIGURA 1: Delineamento experimental em esquema fatorial utilizado para avaliação dos efeitos da temperatura e da proporção de adição de lignina kraft nos resíduos de maravalha e painéis com três repetições.

FIGURE 1: Experimental design in factorial scheme used to assess the effects of temperature and the proportion of the kraft lignin addiction in the shaving and board wastes with three replications.

nos dois diferentes resíduos sobre a propriedade mecânicas dos briquetes avaliados e suas repetições (R).

Para as propriedades mecânicas, foi usado o delineamento inteiramente casualizado em esquema fatorial, com três fatores: temperatura de compactação $\left(60{ }^{\circ} \mathrm{C}, 75^{\circ} \mathrm{C}\right.$ e $\left.90{ }^{\circ} \mathrm{C}\right)$, tipo de resíduo (partículas de maravalha, partículas de resíduos de painéis) e 4 proporções $(0,20,40,60 \%)$ de adição de lignina kraft, com 3 repetições, totalizando 24 tratamentos e 72 unidades amostrais. Os resultados foram interpretados com auxílio de análise de variância (ANOVA) a 95\% de probabilidade. Para os efeitos de proporção e temperatura foi usada a análise de regressão com ajustes e modelos linear simples e quadrático. Para o fator resíduo foi realizado o teste de Tukey $(\alpha=5 \%)$. Utilizou-se o programa SISVAR para a realização da análise.

\section{RESULTADOS E DISCUSSÇÃO}

Verificou-se que as interações temperatura de compactação (TEMP) x adição de lignina (PROP) e tipo de resíduo (RES) x adição de lignina (PROP) foram significativas a 5\% de probabilidade de acordo com a Tabela 1. Desta forma procedeu-se o desdobramento das interações.

Na Tabela 2 encontra-se o resumo da análise de variância para o desdobramento de temperatura, nas proporções de adição de lignina kraft e proporção de adição de lignina kraft dentro de temperatura para a carga de ruptura dos briquetes. Observou-se que a interação foi não significativa somente para as 
TABELA 1: Resumo da análise de variância para resistência mecânica (ResM).

TABLE 1: Summary of the variance analysis for mechanical strength.

\begin{tabular}{|c|c|c|}
\hline \multirow{2}{*}{ FV } & \multirow{2}{*}{ GL } & Quadrado médio \\
\hline & & ResM \\
\hline TEMP (T) & 2 & $34,984968 \mathrm{~ns}$ \\
\hline RES(R) & 1 & $1166,364501 *$ \\
\hline PROP(P) & 3 & $15131,067505 *$ \\
\hline $\mathrm{T} X \mathrm{R}$ & 2 & $134,493901 \mathrm{~ns}$ \\
\hline $\mathrm{T} X \mathrm{P}$ & 6 & $536,254005 *$ \\
\hline R X P & 3 & $227,755605 *$ \\
\hline TXRXP & 6 & $48,293172 \mathrm{~ns}$ \\
\hline Resíduo & 48 & 46,167557 \\
\hline Total & 71 & \\
\hline Média & & 49,3656944 \\
\hline $\mathrm{CV}(\%)$ & & 13,76 \\
\hline
\end{tabular}

Em que: $\mathrm{FV}=$ fonte de variação, $\mathrm{GL}=$ grau de liberdade; $*$ e ns $=$ significativo, a 5\% de probabilidade e não significativo, respectivamente.

proporções de 0 e $60 \%$ de lignina kraft dentro de temperatura. Isso indica que elevadas proporções de lignina kraft adicionada aos resíduos de madeira para produção de briquetes podem não contribuir para ganhos tecnológicos.

TABELA 2: Resumo da análise de variância do desdobramento de temperatura dentro de cada proporção de adição de lignina kraft nos briquetes e proporção de adição de lignina kraft dentro de temperatura para resistência mecânica.

TABLE 2: Summary of the variance analysis of the unfolding temperature inside of each addition proportion of kraft lignin in the briquettes and the proportion of the adding kraft lignin inside temperature for mechanical strength.

\begin{tabular}{|c|c|c|}
\hline \multirow{2}{*}{ FV } & \multirow{2}{*}{ GL } & Quadrado médio \\
\hline & & ResM \\
\hline TEMP/0 & 2 & $20,279756 \mathrm{~ns}$ \\
\hline TEMP/20 & 2 & $766,823339 *$ \\
\hline TEMP/40 & 2 & $55,522172 *$ \\
\hline TEMP/60 & 2 & $801,121717 \mathrm{~ns}$ \\
\hline Resíduo & 48 & $46,167557 *$ \\
\hline \multirow{2}{*}{$\mathrm{FV}$} & \multirow{2}{*}{ GL } & Quadrado médio \\
\hline & & ResM \\
\hline $\mathrm{PROP} / 60$ & 3 & $3808,291033 *$ \\
\hline PROP/75 & 3 & $6075,277871 *$ \\
\hline PROP/90 & 3 & $6320,006611 *$ \\
\hline Resíduo & 48 & 46,167557 \\
\hline
\end{tabular}


Verificou-se, também, que houve diferença significativa em nível de 5\% de probabilidade para as proporções de adições de lignina na composição dos briquetes em função da temperatura de compactação, na carga de ruptura máxima (Tabela 2 ).

Na Figura 2 observa-se a avaliação da carga máxima de ruptura dos briquetes, produzidos, em função da temperatura de compactação, adição de lignina kraft e resíduo utilizado.

A carga máxima de ruptura é uma análise que interfere, de forma significativa, na qualidade de briquetes, pois está relacionada com seu transporte e armazenamento e de certa forma com a durabilidade do mesmo.

De acordo com a Figura 2A, observa-se que para os briquetes produzidos com $60 \%$ de lignina na sua composição, o aumento da temperatura de compactação ocasionou uma perda de resistência. Para os briquetes produzidos com 20 e $40 \%$ de lignina na composição, um incremento da temperatura promoveu o aumento da carga de ruptura dos briquetes, sendo menos evidente para a proporção de $40 \%$ de adição de lignina kraf. Isto evidencia que a lignina kraft apresentou baixas temperaturas de plasticização. Payne (1978) incrementou a resistência de pellets com a adição de 2,5 \% de lignina na composição, obtendo valores
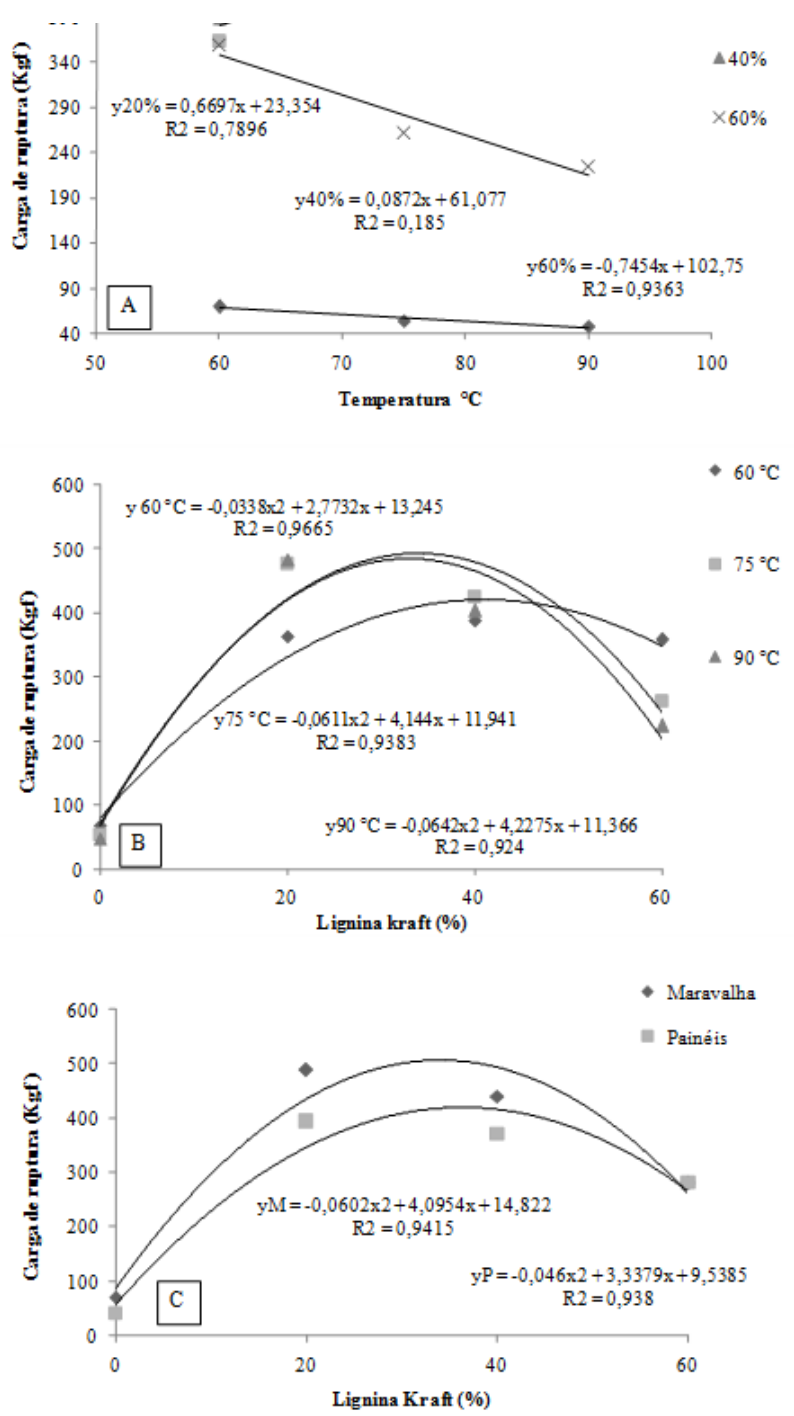

FIGURA 2: Carga máxima de ruptura dos briquetes produzidos com diferentes resíduos em função da temperatura de compactação (A), da adição de lignina kraft (B) e dos resíduos utilizados (C).

FIGURE 2: Maximum tensile load of the briquettes produced with different waste in due of the compaction temperature (A) of the kraft lignin addition (B) and utilized wasted (C). 
médios de carga de ruptura de 16,92 kgf, enquanto os produzidos sem a lignina obtiveram valores médios de $13,39 \mathrm{kgf}$, ou seja, $27 \%$ de incremento na resistência, devido à adição de 2,5\% de lignina. Resistência adequada é importante para a movimentação e transporte dos materiais lignocelulósicos compactados, pois evita-se esmagamento no transporte e a quebra durante a queda (NOVAKOVA; BROZEK, 2008).

No presente trabalho, foram utilizadas temperaturas entre $60^{\circ} \mathrm{Ce} 90^{\circ} \mathrm{C}$, pois em testes preliminares, verificou-se que temperaturas de compactação mais elevadas acarretaram o escoamento excessivo da lignina kraft, impedindo a união com as partículas de maravalha e resíduos de painéis. De acordo com a Figura 2A observou-se que adições de lignina krat acima de $40 \%$ acarretaram perda de qualidade dos briquetes, que foi intensificada com o uso da temperatura em $75^{\circ} \mathrm{C} \mathrm{e} 90^{\circ} \mathrm{C}$. Os briquetes confeccionados na temperatura de $60{ }^{\circ} \mathrm{C}$ apresentaram carga semelhante de ruptura na proporção de 20 e $60 \%$ de adição de lignina kraft e valores próximos e superiores na proporção de $40 \%$.

$\mathrm{Na}$ Figura 2B observou-se que os maiores valores de carga de ruptura foram observados para os briquetes produzidos na temperatura de 60 e $75{ }^{\circ} \mathrm{C}$ com $20 \%$ de adição de lignina kraft, tendo a sua resistência reduzida com maiores adições de lignina kraft.

De acordo com a Figura $2 \mathrm{C}$ os briquetes produzidos com resíduos de maravalha apresentaram maiores cargas de ruptura em relação aos produzidos com resíduos de painéis, exceto para a proporção de $60 \%$ de adição de lignina kraft. Os maiores valores de carga de ruptura foi observado para os briquetes produzidos com $20 \%$ de lignina kraft.

Na Tabela 3 encontra-se a análise de variância para o desdobramento de resíduos, nas proporções de adição de lignina kraft e proporções de adição de lignina kraft em função do resíduo para a carga de ruptura dos briquetes.

Observou-se que a interação resíduos proporção foi não significativa somente para as proporções de 0 e $60 \%$ de lignina kraft.

Para o desdobramento da adição de lignina krat em função do resíduo, a carga máxima de ruptura dos briquetes, foi constatado efeito significativo em relação ao tipo de resíduos, procedeu-se então a análise de regressão.

Vale salientar que a adição da lignina kraft à composição da matéria prima utilizada para a produção

TABELA 3: Resumo da análise de variância do desdobramento de resíduos dentro de proporção de adição e de lignina kraft e proporção dentro de resíduo para resistência mecânica dos briquetes.

TABLE 3: Summary of variance analysis for the unfolding wastes inside of proportion kraft lignin addition and proportion inside of waste for mechanical strength.

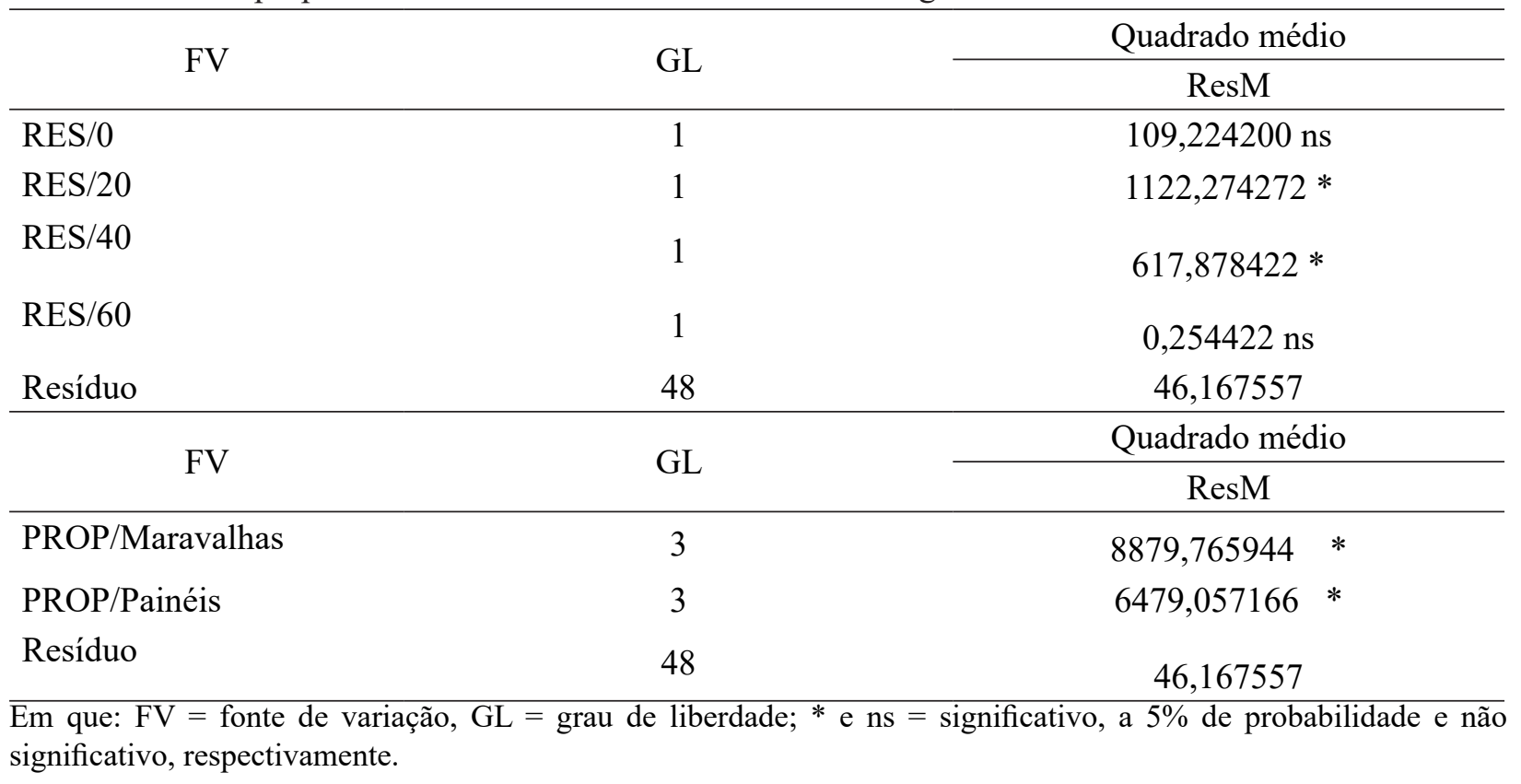


dos briquetes visa o aumento da resistência mecânica e aumento do poder calorifico, principalmente, quando se pretende trabalhar com materiais lignocelulósicos em temperaturas de compactação abaixo dos níveis ideais para plasticização da lignina presente na biomassa e que apresentam baixo poder calorifico

\section{CONCLUSÕES}

De acordo com o estudo realizado, foi possível concluir que:

Os briquetes com maior resistência mecânica foram os produzidos com maravalha e com adição de $20 \%$ de lignina kraft, nas temperaturas de $75^{\circ} \mathrm{C}$ e $90^{\circ} \mathrm{C}$.

A lignina kraft deve ser adicionada aos briquetes buscando aumento da resistência quando se pretende trabalhar com materiais lignocelulósicos em temperaturas de compactação abaixo dos níveis ideais para plasticização, além de contribuir com o imcremento em poder calorífico.

\section{AGRADECIMENTOS}

Os autores agradecem a CAPES pela concessão da bolsa para o desenvolvimento do estudo e a FAPEMIG por disponibilizar o recurso para a execução do projeto.

\section{REFERÊNCIAS}

ASSOCIAÇÃO BRASILEIRA DE NORMAS TÉCNICAS. NBR 9484: Compensado - Determinação do teor de umidade. Rio de Janeiro, 1986.

ASSOCIAÇÃO BRASILEIRA DE NORMAS TÉCNICAS. ABNT NBR ISO 11093-9: Papel e cartão - Ensaio de tubetes - Determinação da resistência ao esmagamento. Rio de Janeiro, 2009. 3 p.

BELGACEM, N. M.; BLAYO, A.; GANDINI, A. Organosolv lignin a filler in inks, varnishes and paints". Industrial Crops and Products, St Martin d'Hères, France, v. 18, p. 145-153, 2004.

DUNKY, M.; PIZZI, T.; LEEMPUT, M. V. Wood Adhesion and Glued Products. Report on the State of the Art. 1st ed. [s. 1.: s.n.], 2002. 174 p.

GARCÍA, H. F.; MARTÍN, J. F.; RODRÍGUEZ, J. J. Posibilidades de aprovechamiento de la lignina en la industria química. Ingeniería Química, Madrid, p. 249-254, 1984.

GOUVÊA, A. F. G. Produção de briquete a partir da adição da lignina kraft com resíduo da indústria moveleira. 2013. 110 f. Tese (Doutorado em Ciências Florestais) - Universidade Federal de Viçosa, Viçosa, MG, 2013.

KALIYAN, K.; MOREY, R. V. Factors affecting strength and durability of densified biomass products. Biomass \& Bioenergy, Minnesota, v. 33, n. 3, p. 337-359, 2009.

LURII, V. G. Comparative results of the combustion of lignin briquettes and black coal. Solid Fuel Chemistry, Moscow, v. 42, n. 6, p. 342-348, 2008.

NORTHEY, R. A. Low cost uses of lignin in materials and chemicals from biomass. American Chemistry Society, Symposium Series, n. 476, p. 146-175, 1992.

NOVAKOVA, A.; BROZEK, M. Mechanical Properties of Pellets from Sorrel. Czech University of Life Sciences Prague, Faculty of Engineering. Engineering for Rural Development, Jelgava, 2008, p. 29.

PAYNE, J. D. Improving quality of pellets feeds. Milling Feed and Fertilizer, Viena, v. 162, p. 34-41, 1978.

PER TOMANI, N. B.; SVÄRD, S. H.; ÅMAND, L. Pilot-Scale Combustion with lignin as a solid bioful. Tappi Engineering, Pulping and Environmental Conference, Portland, 2008. p. 25-27.

VAZ JUNIOR, S. O Potencial dos coprodutos e dos resíduos da biomassa agroindustrial na química renovável. Informativo CRQ-IV, São Paulo, 2012.

WARNER, J. C.; CANNON, A. S.; DYE, K. M. "Green Chemistry". Environmental Impact Assessment Review, New York, v. 24, p. 775-799, 2004. 